\title{
Tratamento de fraturas acetabulares de duas colunas por dupla via extrapélvica: 3 casos clínicos*
}

\section{Treatment of Both-Column Acetabular Fractures by Double Extrapelvic Approach: 3 Clinical Cases}

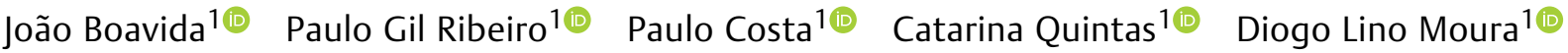 \\ António Figueiredo ${ }^{10}$ \\ ${ }^{1}$ Serviço de Ortopedia, Centro Hospitalar e Universitário de Coimbra \\ EPE, Coimbra, Portugal \\ Endereço para correspondência João Boavida, Mestrado Integrado \\ em Medicina, Serviço de Ortopedia do Centro Hospitalar e \\ Rev Bras Ortop \\ Universitário de Coimbra EPE, Coimbra, Portugal \\ (e-mail: joaoboavida8@gmail.com).
}

\section{Resumo \\ Palavras-chave \\ - fraturas ósseas \\ - acetábulo \\ - pelve}

As fraturas de duas colunas do acetábulo segundo a classificação de Letournel são das mais comuns em frequência, indicação e complexidade cirúrgica. Estas resultam essencialmente de mecanismos de compressão lateral e caracterizam-se por originarem um acetábulo desconectado do esqueleto axial. O seu tratamento cirúrgico pode incluir: abordagem anterior ou posterior isolada; combinadas, no mesmo tempo cirúrgico ou não; ou abordagens alargadas. Os autores apresentam outra opção cirúrgica com associação de via de Kocher-Langenbeck e abordagem da crista ilíaca simultânea e no mesmo posicionamento (decúbito lateral) com base nos três primeiros casos clínicos realizados e seus resultados clínicos e imagiológicos. Para além da apresentação dos casos, é feita uma descrição dos três fragmentos característicos deste tipo de fraturas acetabulares, da via de abordagem e da sequência de redução realizada. Pelos resultados obtidos e vantagens associadas, os autores acreditam que a adição da abordagem da crista ilíaca à via de Kocher-Langenbeck pode ser uma opção muito atrativa a ter em conta no tratamento cirúrgico de fraturas de duas colunas do acetábulo devidamente selecionadas.

Fractures of two columns of the acetabulum according to the Letournel classification are among the most common in frequency, indication and surgical complexity. These are mainly the result of lateral compression mechanisms and are characterized by originating a disconnected acetabulum from the axial skeleton. Its surgical treatment may include: isolated anterior or posterior approach; combined, at the same surgical time or not; or broad approaches. The authors present another surgical option with
Trabalho desenvolvido no Serviço de Ortopedia do Centro Hospitalar e Universitário de Coimbra, Faculdade de Medicina da Universidade de Coimbra, Coimbra, Portugal recebido

08 de Novembro de 2020

aceito

17 de Dezembro de 2020
DOI https://doi.org/

10.1055/s-0041-1729934. ISSN 0102-3616. (c) 2021. Sociedade Brasileira de Ortopedia e Traumatologia. All rights reserved.

This is an open access article published by Thieme under the terms of the Creative Commons Attribution-NonDerivative-NonCommercial-License, permitting copying and reproduction so long as the original work is given appropriate credit. Contents may not be used for commercial purposes, or adapted, remixed, transformed or built upon. (https://creativecommons.org/ licenses/by-nc-nd/4.0/)

Thieme Revinter Publicações Ltda., Rua do Matoso 170, Rio de Janeiro, RJ, CEP 20270-135, Brazil 
association of the Kocher-Langenbeck pathway with the iliac crest approach simultaneously and in the same positioning (lateral decubitus) based on the first three clinical cases performed and their clinical and imaging results. In addition to the presentation of the cases, a description of the three characteristic fragments of this type of acetabular fractures, the approach pathway, and the reduction sequence performed are made. From the results obtained and the associated advantages, the authors believe that the addition of the iliac crest approach to the Kocher-Langenbeck pathway may be a very attractive option to consider in the surgical treatment of properly selected fractures of two columns of the acetabula.

\section{Introdução}

As fraturas do acetábulo constituem 2 a $8 \%$ de todas as fraturas e resultam majoritariamente de traumatismos de alta energia. ${ }^{1,2}$ Desde 1964, com Judet e Letournel, se acredita que a osteossíntese de fraturas do acetábulo desviadas garante melhores resultados do que o tratamento conservador. ${ }^{2}$ As fraturas de duas colunas do acetábulo, segundo a classificação de Letournel, são um dos três tipos mais comuns em frequência, indicação e complexidade cirúrgica, sendo o desvio inicial e a presença de fragmentos intra-articulares os maiores preditores de um resultado menos favorável, e a redução mais perto da anatómica o principal fator preditor de bom resultado. ${ }^{1-7}$ Tais fraturas resultam essencialmente de mecanismos de compressão lateral, sendo o único tipo de fratura com atingimento das duas colunas cujos traços de fratura se encontram acima do acetábulo, originando um acetábulo flutuante, sem qualquer porção conectada ao esqueleto axial. ${ }^{1,4,8}$ Nas opções cirúrgicas, incluem-se: abordagem anterior com redução e fixação indiretas da coluna posterior; abordagem anterior seguida de abordagem posterior, quer no mesmo tempo cirúrgico (o que implica reposicionamento), quer em tempos cirúrgicos distintos; abordagem posterior seguida de abordagem anterior, conduta rara, dado que habitualmente se deve começar pela via anterior; abordagem iliofemoral alargada, cada vez mais desaconselhada pelas suas elevadas extensão e agressividade; e abordagem posterior com redução e fixação indiretas da coluna anterior. ${ }^{2,4,6,8,9} \mathrm{Na}$ linha desta última opção, os autores apresentam uma opção cirúrgica (dupla abordagem simultânea e em decúbito lateral: via de KocherLangenbeck e abordagem da crista ilíaca) com base nos três primeiros casos clínicos e nos seus resultados clínicos e imagiológicos

\section{Relato de Caso}

\section{Caso 1}

Paciente do sexo feminino, 59 anos, vítima de atropelamento, chega ao nosso centro com traumatismo da hemibacia direita. O estudo imagiológico permitiu diagnosticar uma fratura das duas colunas do acetábulo com envolvimento do ilíaco posterior e com luxação central da cabeça femoral. Foi aplicada tração esquelética nos côndilos femorais com peso correspondente a $10 \%$ do peso corporal, e a paciente foi internada. Após planeamento cirúrgico, avançou-se para o tratamento definitivo: redução e osteossíntese do ilíaco com duas placas e redução da fratura da coluna posterior e osteossíntese com placa pela via de Kocher-Langenbeck combinada com abordagem da crista ilíaca. Para melhor acesso ao componente anterior da fratura, fixada com duas placas, houve necessidade de osteotomia do grande trocânter. 0 protocolo pós-operatório utilizado foi o comumente utilizado na instituição para este tipo de lesões e comum aos casos seguintes: início precoce de mobilização e reforço muscular em função da evolução cicatricial, descarga do membro operado durante as primeiras 6 semanas e carga parcial progressiva durante as 6 semanas seguintes, com carga total autônoma permitida aos 3 meses. Com 3 anos de evolução, a paciente encontra-se satisfeita, sem dores, com marcha autónoma e mobilidade articular semelhante à anca contralateral, com um Harris Hip Score de 84 pontos (-Figura 1).

\section{Caso 2}

Paciente do sexo masculino, 49 anos, deu entrada no serviço de urgência após queda de 7 metros de altura com traumatismo da hemibacia direita e traumatismo torácico, da qual resultaram fraturas de arcos costais com pneumotórax associado e fratura das duas colunas do acetábulo com extensão alta para o ilíaco e com luxação central da cabeça. Após avaliação e estabilização multidisciplinar, optou-se por redução fechada da luxação central sob anestesia com controle fluoroscópico e aplicação de tração esquelética no fêmur distal. Após estabilização clínica, procedeu-se ao tratamento cirúrgico definitivo: redução aberta e osteossíntese pela via de Kocher-Langenbeck combinada com abordagem da crista ilíaca. Foi cumprido o protocolo de reabilitação e, aos 9 meses de evolução, o paciente encontra-se satisfeito e a fazer marcha sem apoio ou limitação para as atividades de vida diárias, com um Harris Hip Score de 95 pontos (-Figura 2).

\section{Caso 3}

Paciente do sexo masculino, 69 anos, referenciado de outra instituição após queda de 3 metros de altura da qual resultou fratura das duas colunas do acetábulo esquerdo com extensão alta para o ilíaco. Após estabilização clínica, estudo imagiológico completo e planeamento, procedeu-se ao tratamento cirúrgico definitivo: redução aberta e osteossíntese com placa da coluna posterior, redução indireta da 

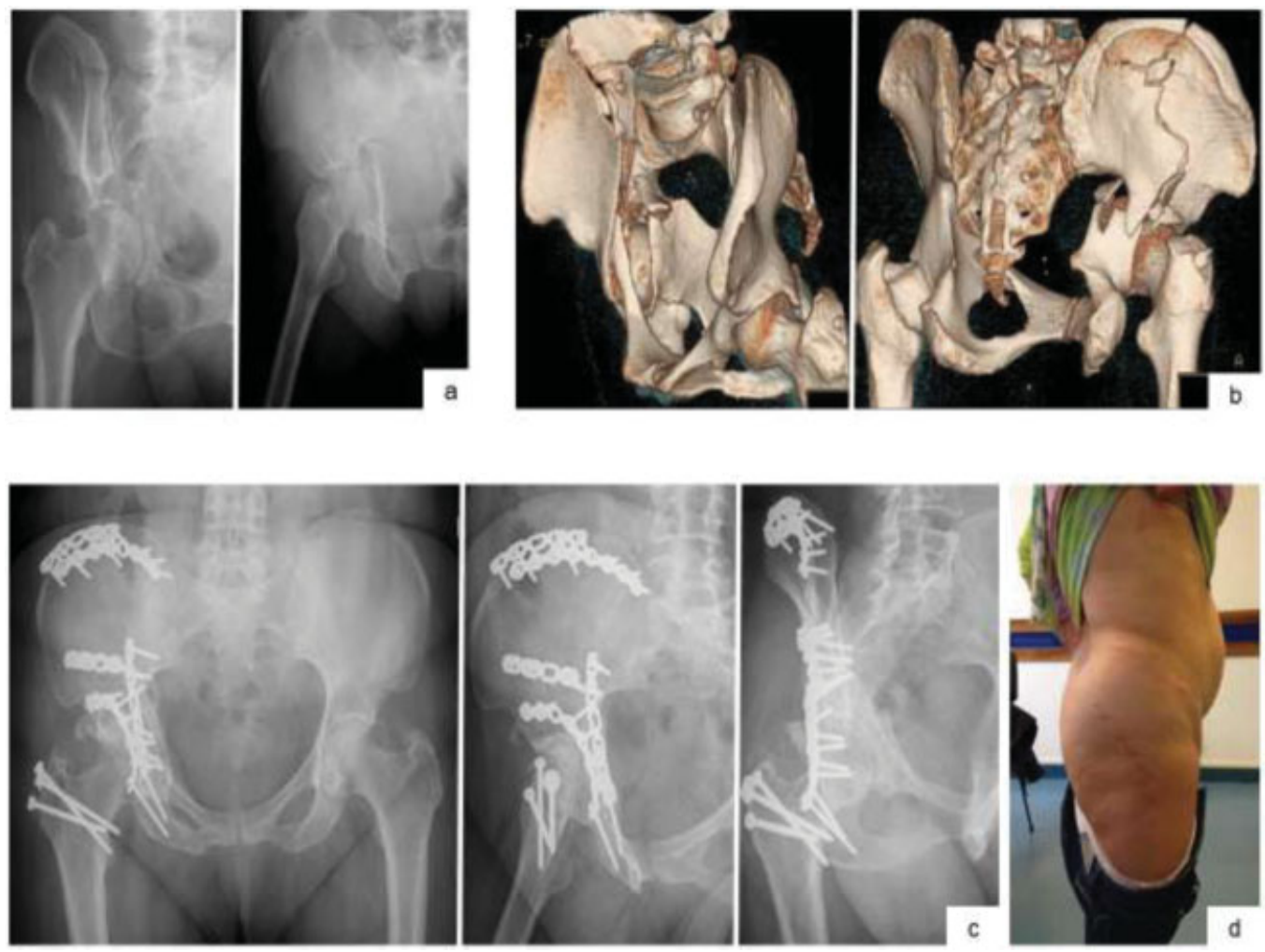

Fig. 1 Imagens de radiografias nas incidências obturadora e alar, com típico sinal do esporão na primeira imagem (a) Reconstruções 3D (b) Controle radiológico nas incidências inlet, alar e obturadora ao $3^{\circ}$ ano de pós-operatório(c) Fotografia da paciente com cicatrizes das vias utilizadas (d).

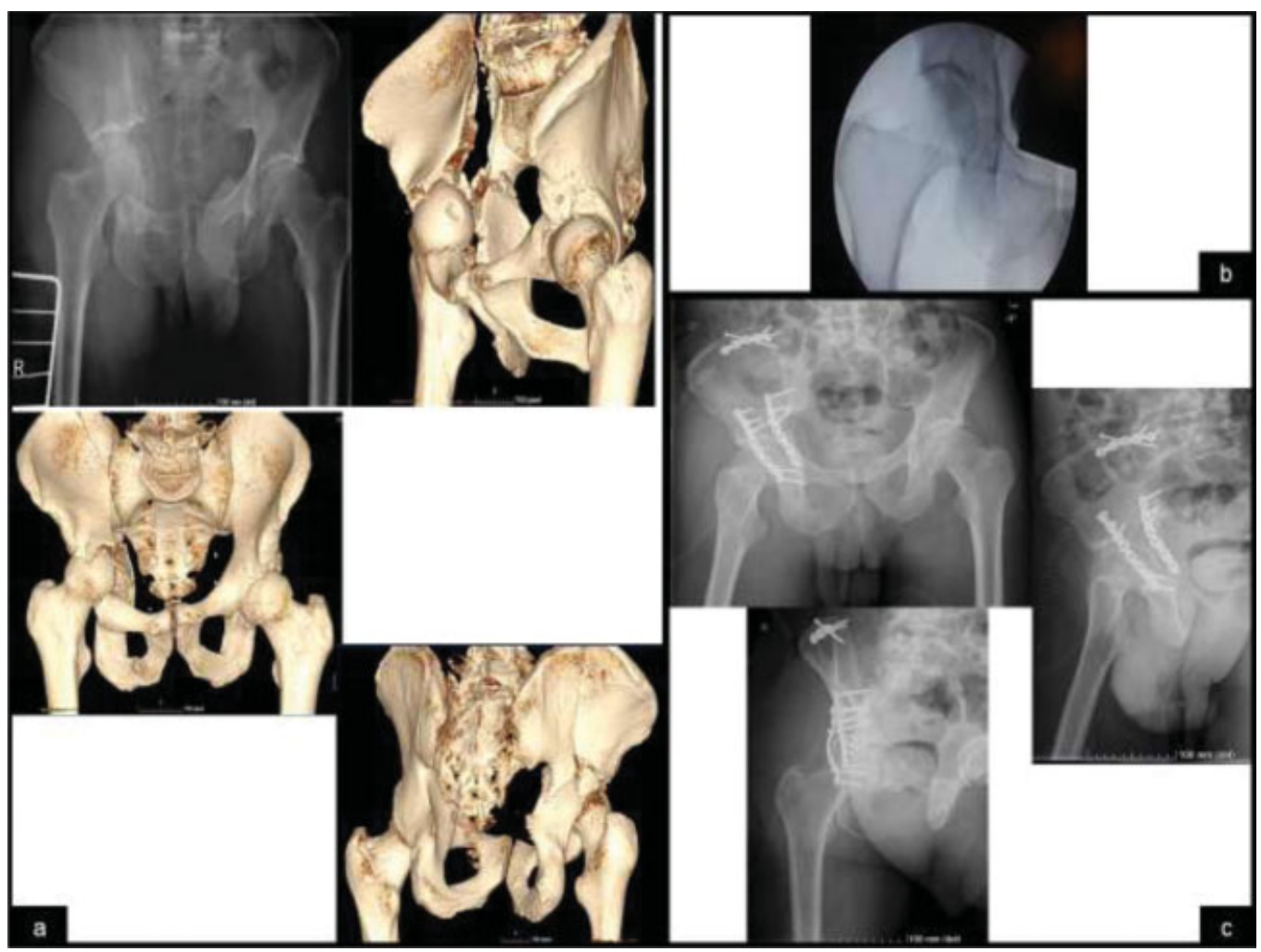

Fig. 2 Imagens de radiografia anteroposterior da bacia e imagens de TAC 3D para melhor caracterização da fratura de duas colunas do acetábulo direito com extensão alta para o ilíaco (a) Imagem intraoperatória após manobras de redução da luxação central (b) Controle radiológico nas incidências anteroposterior da bacia, alar e obturadora da anca direita no pós operatório, com redução aceitável e ausência de parafusos intra-articulares. 


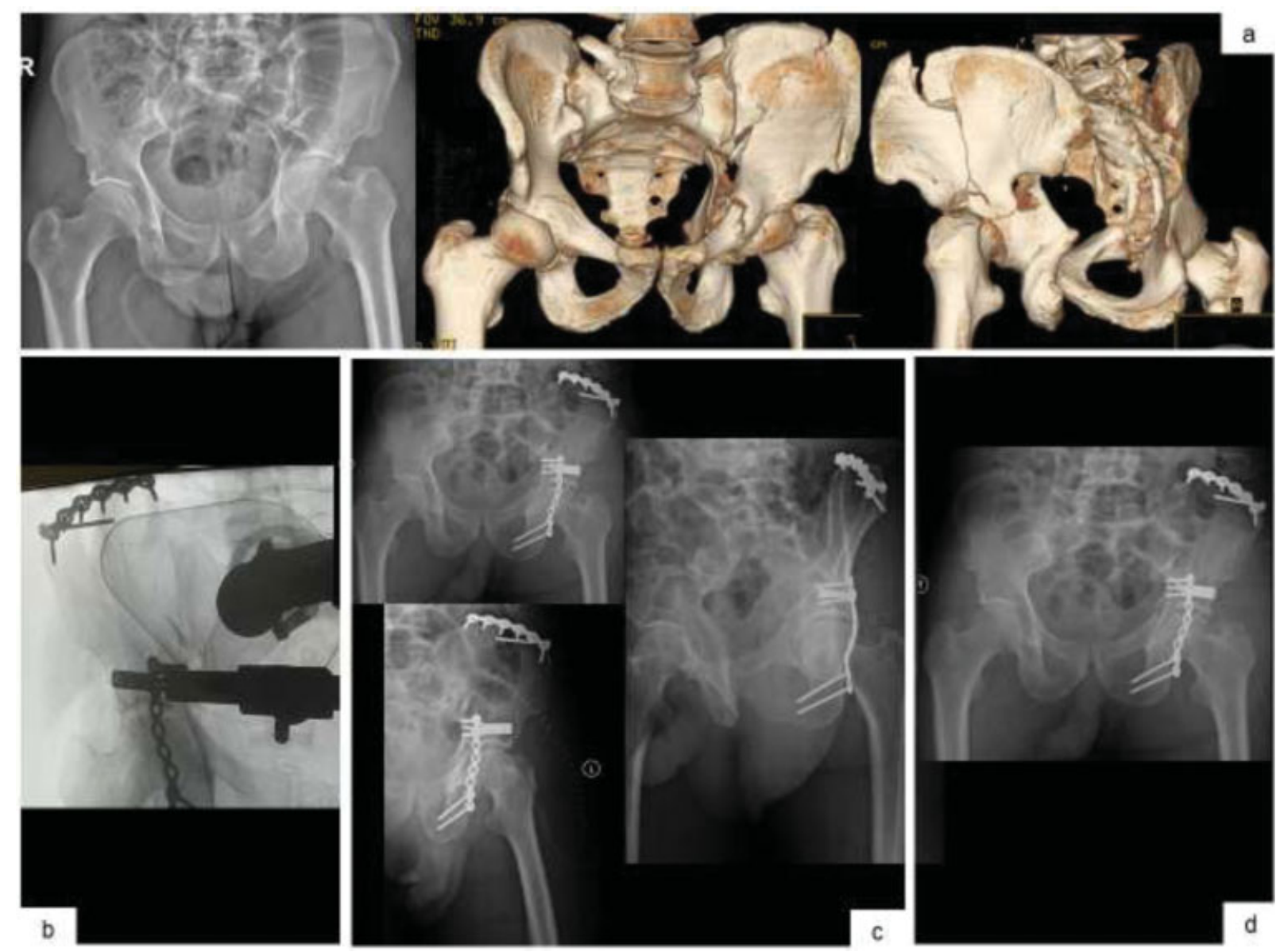

Fig. 3 Imagens de radiografia anteroposterior da bacia e imagens de TAC 3D para melhor caracterização da fratura de duas colunas do acetábulo esquerdo com extensão alta para o ilíaco (a) Imagem de controle intraoperatório (b) Controle radiológico nas incidências anteroposterior da bacia, alar e obturadora da anca esquerda no pós-operatório imediato (c) Radiografia anteroposterior da bacia ao $3^{\circ}$ mês (d).

coluna anterior e redução aberta da fratura do ilíaco e fixação com duas placas e parafuso em compressão pela via de Kocher-Langenbeck combinada com abordagem da crista ilíaca. Com 7 meses de evolução, o paciente encontra-se a fazer marcha autónoma e sem dores, com um Harris Hip Score de 91 pontos (-Figura 3).

\section{Discussão}

As fraturas de duas colunas são das fraturas acetabulares mais comuns e caracterizam-se por serem complexas e desafiantes do ponto de vista cirúrgico. ${ }^{1-5,7}$ Os três casos clínicos apresentados representam muito daquilo que as caracteriza: mecanismos de lesão de alta energia, vetor de energia essencialmente lateral e junção dos traços de fratura acima do acetábulo, que fica desconectado do esqueleto axial. A decisão e planeamento cirúrgico de pacientes com este tipo de lesão exige experiência nas diferentes abordagens acetabulares e implicam um profundo entendimento do mecanismo de lesão e desvio dos fragmentos. É essencial uma avaliação pormenorizada do paciente, das lesões associadas, e estudo imagiológico completo incluindo reconstruções 3D de tomografia axial computarizada (TAC). Este planeamento é o passo mais importante para o sucesso global. Os autores apresentam três casos clínicos nos quais as características da lesão ditaram a opção tomada. A - Figura 4 permite exemplificar o posicionamento e a abordagem, e a -Figura 5 é representativa da sequência sugerida de redução e fixação. Na opinião dos autores, as

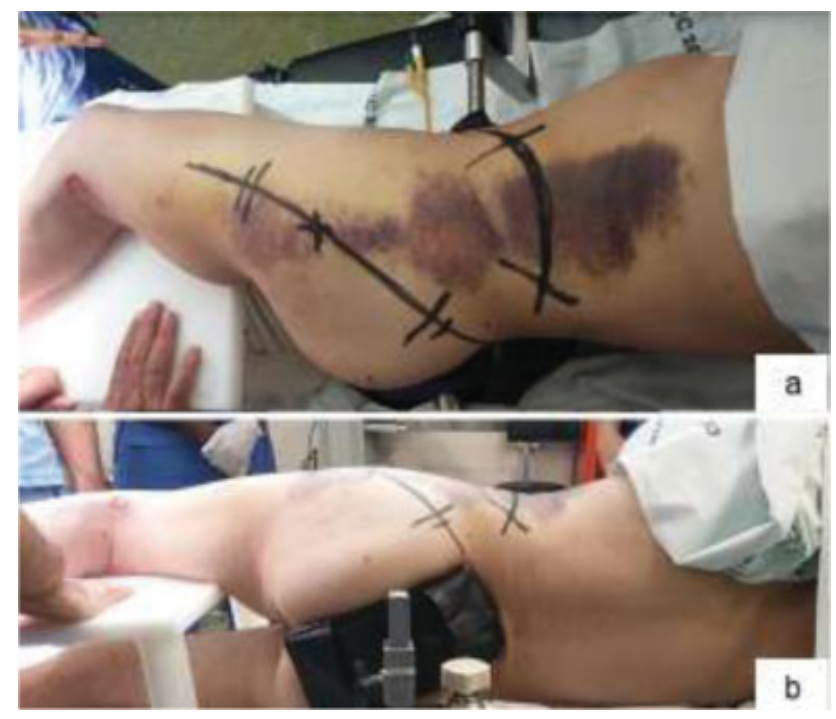

Fig. 4 Posicionamento do paciente em decúbito lateral e marcação prévia das incisões (a) Vista posterior do posicionamento, sendo neste plano que entra o intensificador de imagem (b). 

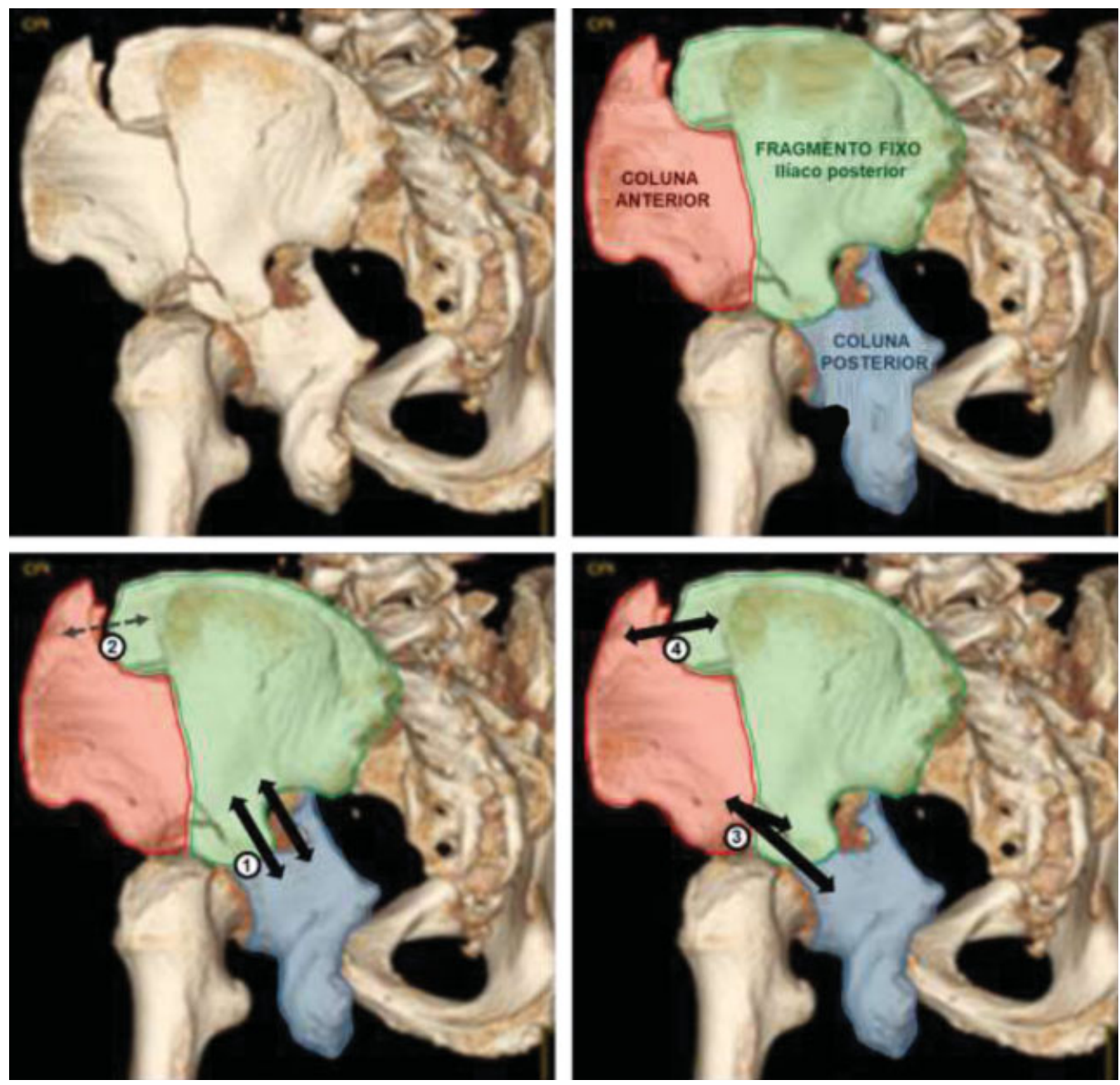

Fig. 5 Em cima: Os 3 fragmentos principais das fraturas de duas colunas do acetábulo: coluna anterior, coluna posterior e ilíaco posterior. Este último fica ligado ao esqueleto axial e engloba a chanfradura ciática; a coluna anterior (com desvio essencialmente medial) e a coluna posterior (com desvio medial e posterior) ficam livres, com o seu desvio mais facilmente perceptível na incidência obturadora, onde se visualiza a chanfradura ciática in situ lateralmente e 0 desvio medial das colunas e acetábulo (sinal do esporão). (6) Embaixo: ordem sugerida de redução e osteossíntese dos fragmentos por abordagens simultâneas (via de Kocher-Langenbeck e abordagem da crista ilíaca): $1^{\circ}$ - redução e fixação da coluna posterior ao ilíaco posterior; $2^{\circ}$ - redução provisória da porção superior da coluna anterior ao ilíaco posterior ; $3^{\circ}$ - redução da parte distal da coluna anterior e sua fixação ao ilíaco posterior e/ou à coluna posterior; $4^{\circ}$ - fixação definitiva da porção superior da coluna anterior ao ilíaco posterior.

principais vantagens desta opção são: cirurgia extrapélvica, realizada em um único tempo cirúrgico e posicionamento, sem um potencial de complicações similar ao de uma abordagem alargada. Adicionalmente, em caso de fragmentos intra-articulares ou de fraturas associadas da cabeça femoral, é possível associar luxação segura da anca. Em conclusão, apesar da necessidade de mais estudos prospectivos, randomizados e comparativos, com amostras e tempo de seguimento relevantes, os autores acreditam que a adição da abordagem da crista ilíaca à via de Kocher-Langenbeck pode ser uma opção muito atrativa a ter em conta no tratamento cirúrgico de fraturas de duas colunas do acetábulo com maior desvio da coluna posterior e extensão alta para o ilíaco.

Consentimento livre e esclarecido

O termo de consentimento livre e esclarecido foi obtido dos pacientes para a publicação de dados referentes aos casos.
Conflito de Interesses

Os autores declaram não haver conflito de interesses.

\section{Referências}

1 Tornetta P 3rd. Displaced acetabular fractures: indications for operative and nonoperative management. J Am Acad Orthop Surg 2001;9(01):18-28

2 Briffa N, Pearce R, Hill AM, Bircher M. Outcomes of acetabular fracture fixation with ten years' follow-up. J Bone Joint Surg Br 2011;93(02):229-236

3 Kelly J, Ladurner A, Rickman M. Surgical management of acetabular fractures - A contemporary literature review. Injury 2020; 51(10):2267-2277

4 Matta JM, Merritt PO. Displaced acetabular fractures. Clin Orthop Relat Res 1988;(230):83-97

5 Lichte P, Sellei RM, Kobbe P, Dombroski DG, Gänsslen A, Pape HC. Predictors of poor outcome after both column acetabular fractures: a 30-year retrospective cohort study. Patient Saf Surg 2013;7(01):9

6 Pierannunzii L, Fischer F, Tagliabue L, Calori GM, d'Imporzano M. Acetabular both-column fractures: essentials of operative management. Injury 2010;41(11):1145-1149 
Tratamento de fraturas acetabulares de duas colunas por dupla via extrapélvica Boavida et al.

7 Park KS, Chan CK, Lee GW, Ahn HW, Yoon TR. Outcome of alternative approach to displaced acetabular fractures. Injury 2017;48(02):388-393

8 Yang Y, Zou C, Fang Y. Mapping of both column acetabular fractures with three-dimensional computed tomography and implications on surgical management. BMC Musculoskelet Disord 2019;20(01):255

9 Giannoudis PV, Grotz MR, Papakostidis C, Dinopoulos H. Operative treatment of displaced fractures of the acetabulum. A metaanalysis. J Bone Joint Surg Br 2005;87(01):2-9 